ARTICLE

DOI: $10.1038 / s 41467-018-03965-8$

\title{
Resonantly enhanced multiple exciton generation through below-band-gap multi-photon absorption in perovskite nanocrystals
}

Aurora Manzi ${ }^{1,2}$, Yu Tong ${ }^{1,2}$, Julius Feucht ${ }^{1,2}$, En-Ping Yao ${ }^{1,2}$, Lakshminarayana Polavarapu (D) 1,2, Alexander S. Urban (1) ${ }^{1,2} \&$ Jochen Feldmann (i) ${ }^{1,2}$

Multi-photon absorption and multiple exciton generation represent two separate strategies for enhancing the conversion efficiency of light into usable electric power. Targeting below-band-gap and above-band-gap energies, respectively, to date these processes have only been demonstrated independently. Here we report the combined interaction of both nonlinear processes in $\mathrm{CsPbBr}_{3}$ perovskite nanocrystals. We demonstrate nonlinear absorption over a wide range of below-band-gap excitation energies (0.5-0.8 $\left.E_{\mathrm{g}}\right)$. Interestingly, we discover high-order absorption processes, deviating from the typical two-photon absorption, at specific energetic positions. These energies are associated with a strong enhancement of the photoluminescence intensity by up to $10^{5}$. The analysis of the corresponding energy levels reveals that the observed phenomena can be ascribed to the resonant creation of multiple excitons via the absorption of multiple below-band-gap photons. This effect may open new pathways for the efficient conversion of optical energy, potentially also in other semiconducting materials.

\footnotetext{
${ }^{1}$ Chair for Photonics and Optoelectronics, Department of Physics and Center for NanoScience (CeNS), Ludwig-Maximilians-Universität, Amalienstr. 54, 80799 Munich, Germany. ${ }^{2}$ Nanosystems Initiative Munich (NIM), Schellingstr. 4, 80799 Munich, Germany. Correspondence and requests for materials should be addressed to A.M. (email: Aurora.Manzi@physik.uni-muenchen.de) or to J.F. (email: feldmann@lmu.de)
} 
S emiconductors are nowadays the most prominent materials for applications in optoelectronic devices. Harvesting the energy of light and converting it into usable electric energy with the lowest amount of losses is one of the main goals to achieve high efficiency in photovoltaic devices. The two main loss sources are (i) the absorption cutoff for photons with energies below the semiconductor band gap $\left(E_{\mathrm{g}}\right)$ and (ii) the wasted excess energy of photons with energies above the band gap. Below-bandgap photons normally pass the respective semiconductor without being absorbed. Multiple photon excitation (MPE) processes, however, represent a route to access such low-energy photons leading to the generation of one exciton via the simultaneous absorption of a number $(\geq 2)$ of below-band-gap photons whose combined energy is greater than the band-gap energy ${ }^{1}$. On the other side, highly energetic photons do not contribute effectively to the total usable energy in semiconducting devices because their excess energy is typically lost as heat. A possible approach to address this limitation is to generate multiple excitons from one photon, a process known as multiple exciton generation (MEG) or carrier multiplication ${ }^{2-5}$. This process typically requires photons with energies several times that of the band gap and has been shown to work efficiently only in nanocrystals exhibiting strong quantum confinement ${ }^{6-12}$.

Here we investigate the combined action of MPE and MEG on the light-emitting properties of non-quantum-confined metal halide perovskite nanocrystals, a semiconducting material that in recent years has shown its high potential for photovoltaic ${ }^{13,14}$ as well as for light-emitting applications ${ }^{15}$. Although these mechanisms have been studied independently in perovskite nanocrystals ${ }^{16-25}$, here we analyze the possibility to combine them together to further enhance their efficiencies under specific resonant conditions. We find interband photoluminescence (PL) of $\mathrm{CsPbBr}_{3}$ nanocubes (NCs) under MPE for a wide spectral range below the band gap. Strikingly, we observe distinct resonances of the PL intensity for specific excitation wavelengths in the MPE-PL spectra. These correspond to specific energies of the excitation source and can be ascribed to the resonant creation of multiple excitons through MPE, as illustrated in Fig. 1.

\section{Results}

Synthesis and optical characterization. The $\mathrm{CsPbBr}_{3}$ NCs were synthesized using a one-step process based on direct tip sonication of precursor mixtures, according to our previously published work ${ }^{26}$. The purified dispersion of NCs exhibits an absorption spectrum characterized by a sharp absorption onset and a distinct excitonic peak at $500 \mathrm{~nm}$ (see Fig. 2a). The PL spectrum of the NCs deposited on a glass substrate shows a single PL emission peak centered around $\lambda_{\mathrm{x}}=523 \mathrm{~nm}$, corresponding to an energy $E_{\mathrm{x}}=h v_{\mathrm{x}}=2.37 \mathrm{eV}$. A spectrometer with an integrating sphere detector was used to measure the absorbance of the NCs deposited on a substrate (Supplementary Fig. 1). The absorbance of the NCs distributed on a glass substrate resembles the absorbance in solution, indicating almost no absorption of the nanocrystals for excitation below the band-gap. The average size of the cubes was determined from transmission electron microscopy (TEM) images to be in the range of $10-15 \mathrm{~nm}$ (Fig. 2b). These sizes along with the absorption and PL spectra clearly show that these NCs do not show quantum confinement, but have already bulk-like properties $^{27,28}$. Moreover, from the TEM and the scanning electron microscopy (SEM) analysis (Fig. 2c), we observe that the NCs are mostly ordered on the substrate leading to a superlatticelike arrangement.

Below-band-gap optical excitation. By studying the emitting properties of the nanocrystals using an excitation source with below-band-gap energy photons, we observe that the $\mathrm{CsPbBr}$ NCs show PL emission for a wide range of excitation wavelengths (see inset in Fig. 3a). The excitation was provided by a focused supercontinuum laser (repetition rate $78 \mathrm{MHz}$ ) with 15 ps pulses tunable from 680 to $1080 \mathrm{~nm}$. For each excitation energy we observe PL centered around $523 \mathrm{~nm}$, indicating almost no shift with respect to the PL measured using above-band-gap excitation. Since the linear absorption of the NCs is negligible in this wavelength range, the absorption processes must be of nonlinear origin.

While the spectral shape of the PL emission does not change with wavelength, the PL intensity depends strongly on the excitation wavelength (see Fig. 3a). There is a clear onset of the PL intensity for an excitation wavelength around $\lambda_{2}=1030 \mathrm{~nm}$ (photon energy $h v_{2}=1.20 \mathrm{eV} \simeq 0.50 E_{\mathrm{g}}$ ), with the intensity generally increasing as the excitation wavelength is decreased. This onset can be associated to the minimum excitation energy required for two photons to overcome the band gap via MPE. A local maximum is reached at $680 \mathrm{~nm}$, after which the PL intensity sharply drops to nearly zero. Even more interestingly, the MPEPL spectrum does not increase monotonically, but rather exhibits two distinct peaks located at excitation wavelengths of $\lambda_{3}=790$ $\mathrm{nm}$ and $\lambda_{4}=700 \mathrm{~nm}$ (photon energies $h v_{3}=1.57 \mathrm{eV} \simeq 0.66 E_{\mathrm{g}}$ and $h v_{4}=1.77 \mathrm{eV} \simeq 0.75 E_{\mathrm{g}}$, respectively). These peaks are associated with a PL intensity which is several orders of magnitude higher $\left(10^{3}\right.$ and $10^{5}$, respectively) than the signal detected in the vicinity of $\lambda_{2}$. For clarity, the positions of the observed peaks in the MPE-PL spectrum are also marked with black arrows in the absorbance spectrum in Fig. 2.

Interestingly, these particular energies perfectly match the energy of multiples of the exciton energy $h v_{x}$, suggesting the possible connection between multiple photon absorption and the resonant generation of multiple excitons. In fact, three photons of energy corresponding to the excitation wavelength $\lambda_{3}$ have the exact energy required to generate two excitons in the NCs. In the same way, four photons of energy associated to the the excitation wavelength $\lambda_{4}$ correspond to the energy necessary to generate three excitons. Analogously, two photons of energy related to the excitation wavelength $\lambda_{2}$ exactly equal the exciton
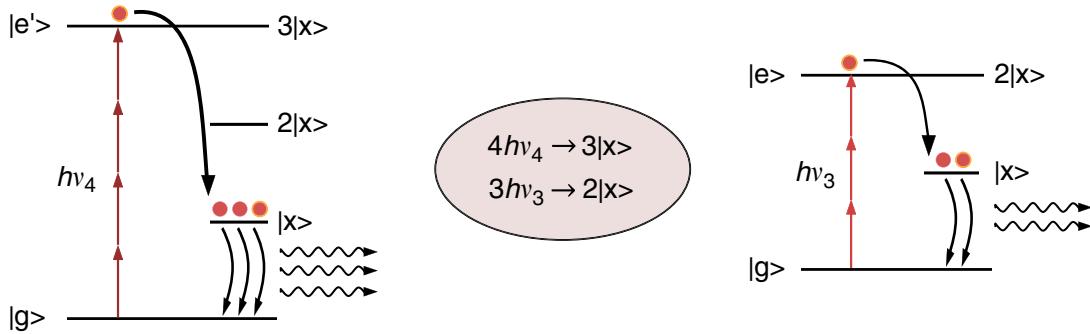

Fig. 1 Schematic of the combined MPE-MEG mechanism. Multiple photon excitation with below-band-gap photons of energies $h \nu_{4}$ and $h \nu_{3}$ resonantly matching the energy levels $3 \mid x>$ and $2 \mid x>$ associated to multiple exciton generation 
a

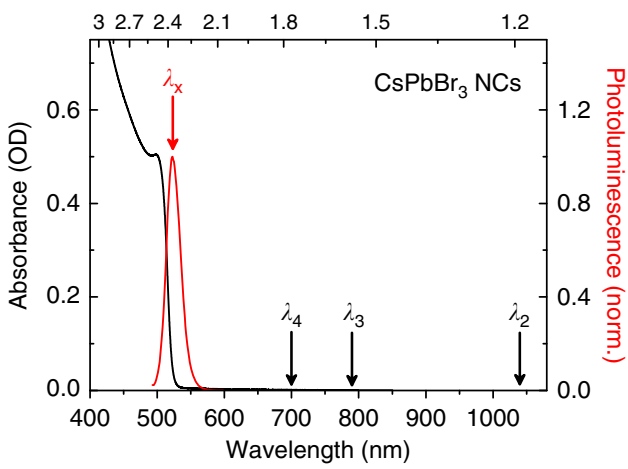

b

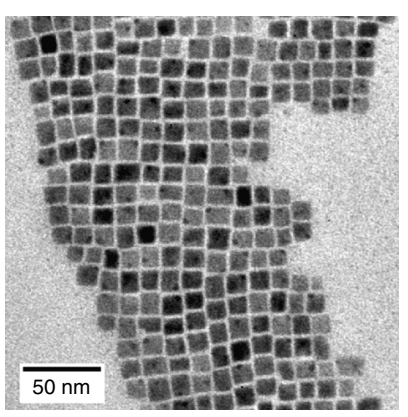

C

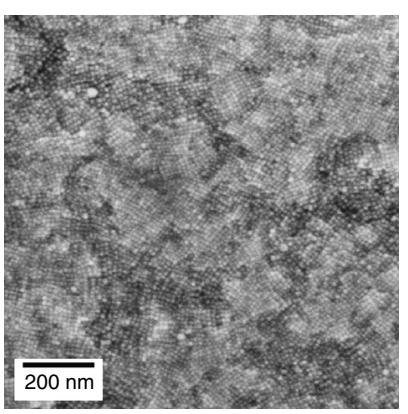

Fig. 2 Optical and morphological characterization of the $\mathrm{CsPbBr}_{3}$ nanocubes. a Absorbance (left axis) spectra of $\mathrm{CsPbBr}_{3}$ nanocubes in hexane and normalized photoluminescence (right axis) of $\mathrm{CsPbBr}_{3}$ nanocubes on substrates, excitation wavelength $480 \mathrm{~nm}$. The arrows indicate, respectively, the position of the photoluminescence emission $\lambda_{x}$, and the excitation wavelengths $\lambda_{4}$, $\lambda_{3}$ and $\lambda_{2}$ associated to the observed resonances. $\mathbf{b}$ TEM image of $\mathrm{CsPbBr}_{3}$ nanocubes, scale bar $50 \mathrm{~nm}$. c SEM image of a $\mathrm{CsPbBr}_{3}$ nanocube film showing the superlattice-like arrangement, scale bar $200 \mathrm{~nm}$

energy $E_{\mathrm{x}}$. No multiple exciton generation is therefore expected in correspondence to $\lambda_{2}$, explaining the absence of an associated peak in the MPE-PL spectrum.

To exclude the possibility that the observed resonances are due to an effect only observable in $\mathrm{CsPbBr}_{3} \mathrm{NCs}$, we have performed analogous measurements on $\mathrm{CsPbI}_{3}$ NCs (Supplementary Fig. 3 and 4 ). These were fabricated through an ion exchange step from the originally synthesized $\mathrm{CsPbBr}$ NCs, preserving their size and geometry (see Supplementary Fig. 3 for more details). In these NCs, we again observe clear resonances in the PLE spectra. Their spectral positions again align with multiples of the exciton energy $\left(E_{\mathrm{x}}=1.81 \mathrm{eV}\right)$, i.e., they are red-shifted with respect to those observed in the $\mathrm{CsPbBr}_{3}$ system. These results support the notion that the proposed mechanism is not a specific property of $\mathrm{CsPbBr}{ }_{3}$ NCs.

Order of the absorption processes. For an absorption process involving $p$ photons, the intensity of the $\mathrm{PL}, I_{\mathrm{PL}}$, is proportional to the excitation intensity to the power of $p,\left(I_{\mathrm{exc}}\right)^{p}$, with $p$ being the order of the process. For $p=1$ this accounts to the regular linear absorption, while for $p>1$ the process corresponds to a multiple photon absorption process via $p-1$ virtual states. Thus, by measuring the PL intensity in dependence of the excitation intensity and applying a power law fit, one can obtain the order of the process and thus determine the nature of the absorption.

In Fig. $3 \mathrm{~b}$ the integrated PL intensities at the respective excitation wavelengths $\lambda_{4}, \lambda_{3}$ and $\lambda_{2}$ are plotted in dependence of the laser excitation intensity. Fitting power law functions to the data, we find that at $\lambda_{4}$ absorption takes place via a fourth-order process, while at $\lambda_{3}$ the process is of third order and at $\lambda_{2}$ of second order. This is in complete accordance with our interpretation of an MPE process.

Analysis of the energy levels. With the knowledge that 4 and 3 photons are involved in the absorption processes associated to the excitation wavelengths $\lambda_{4}$ and $\lambda_{3}$ respectively, we can determine the energy levels reached by the MPE process and correlate them with the energy necessary to generate multiple excitons. A schematic representation of the combined MPE and MEG processes in the analyzed system is shown in Fig. 4 . When $\lambda_{4}$ is used as the excitation wavelength, the system undergoes 4-photon absorption and reaches the energy level $7.08 \mathrm{eV}$, that is resonant with $3 E_{\mathrm{x}}=7.10 \mathrm{eV}$. Similarly, when photons with wavelength $\lambda_{3}$ are used, a 3-photon absorption process is observed and the photogenerated electrons have an energy of $4.71 \mathrm{eV}$, resonant with $2 E_{\mathrm{x}}$
$=4.73 \mathrm{eV}$. Therefore, the energy matching between the energy level reached through MPE and the level associated to multiples of the exciton energy $E_{\mathrm{x}}$ allows the combined interplay of MEG and MPE. The combination of these processes produces the observed maxima in the PL efficiency for specific values of the excitation energy, which is in agreement with the efficient creation of multiple excitons.

\section{Discussion}

The observation of resonances given by MEG in the MPE-PL of $\mathrm{CsPbBr}$ is realized when the following relation is fulfilled:

$$
p \times h \nu=N \times E_{\mathrm{x}}
$$

where $p$ is the number of photons involved in the MPE, $N$ is the number of excitons created through MEG and $E_{\mathrm{x}}$ is the energy of a single exciton. In order to confirm this theory, we look at MPE at excitation wavelengths away from the observed resonances and focus on the order of the excitation process (Fig. 3c). The PL intensity drops off steeply for excitation wavelengths longer than $1030 \mathrm{~nm}$, corresponding to twice the band-gap energy of the $\mathrm{CsPbBr}_{3} \mathrm{NCs}$. For slightly shorter wavelengths, the PL intensity scales proportionally to $\left(I_{\text {exc }}\right)^{2}$, while for slightly longer wavelengths the PL intensity clearly scales with $\left(I_{\text {exc }}\right)^{3}$ (Supplementary Fig. 5). These values confirm that the drop off region marks the onset of two-photon absorption (2PA), while PL emission for excitation at longer wavelengths requires three photon absorption (3PA). For shorter wavelengths the order of the absorption process remains around 2 or slightly below this value (see Supplementary Fig. 6). Close to the resonance at $\lambda_{3}$ the order jumps up to a value of 3 again, and at $\lambda_{4}$, the order increases to 4 . In between these resonances, the order decreases rapidly to 2 . This suggests that the specific resonances are indeed necessary to promote the higher order MPE. Notably, the PL decreases abruptly to almost zero for excitation wavelengths shorter than $680 \mathrm{~nm}$. At these wavelengths, four photon absorption leads to energies in excess of $7 \mathrm{eV}$, which could possibly induce ionization of the NCs, opening a new non-radiative decay channel.

Upon closer inspection, the peaks in the PL spectrum corresponding to excitation wavelengths in the vicinity of resonances have a characteristic non-symmetrical shape (Fig. 3a). Surprisingly, when photons with energies slightly higher than the resonance energy are used for excitation, the efficiency of the emission drops. This is an indication that the combined MPE-MEG process is particularly favorable when no additional excess energy is involved in the excitation process. 
a
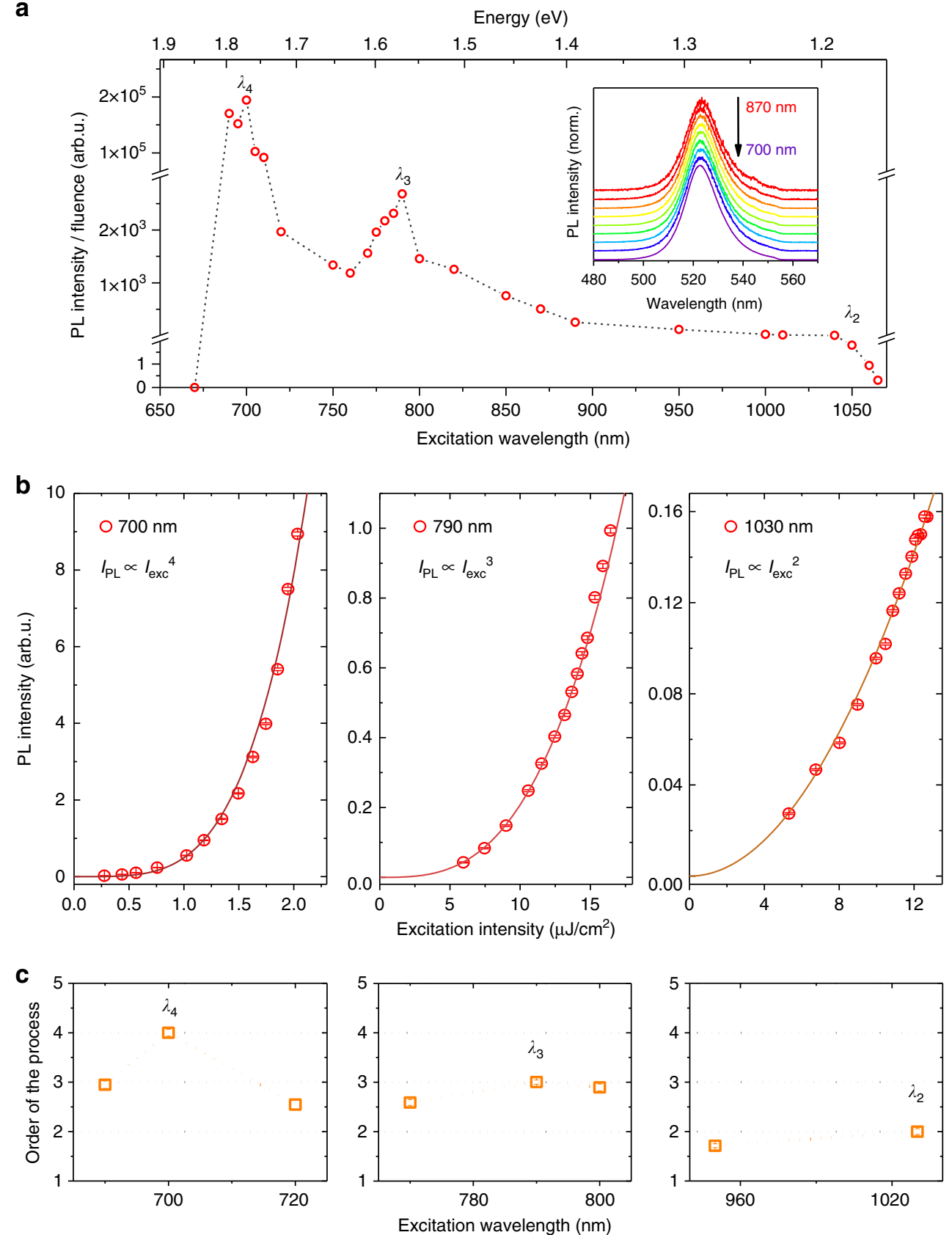

Fig. 3 Nonlinear absorption-induced PL in perovskite NCs. a Photoluminescence intensity of $\mathrm{CsPbBr}_{3} \mathrm{NCs}$ as a function of the below-band-gap excitation wavelength. The PL intensity is normalized by the laser fluence (see Supplementary Fig. 2). The dashed line is a guide to the eye. The inset shows the normalized photoluminescence spectrum for excitation wavelengths varying from 870 to $700 \mathrm{~nm}$ (specifically: 870, 850, 820, 800, 790, 770, 750, 720 and $700 \mathrm{~nm})$. b Integrated photoluminescence intensity as a function of the laser excitation intensity for excitation wavelengths $\lambda_{4}(700 \mathrm{~nm}), \lambda_{3}(790 \mathrm{~nm})$ and $\lambda_{2}(1030 \mathrm{~nm})$. The solid lines represent the respective fit with power functions, from which we can deduce the order of the process: 4 for $\lambda_{4}$ excitation, 3 for $\lambda_{3}$ excitation and 2 for $\lambda_{2}$ excitation. $\mathbf{c}$ Order of the absorption processes as a function of the below-band-gap excitation wavelength

Another striking result is that the resonant MPE-MEG process is absent for such nanocubes under comparable laser excitation intensities when they are dispersed in solution. This suggests that the observed PL enhancement in correspondence with the MEG resonances relies on the close-packed arrangement of the $\mathrm{CsPbBr}_{3} \mathrm{NCs}$ on the substrate. As reported by Trinh et al., MEG in adjacent silicon nanocrystals results in a suppression of the non-radiative Auger recombination and in the creation of longliving multiple excitons ${ }^{29}$. The initial optical excitation possesses a large energy and the related wavefunctions can overlap spatially with those of adjacent nanocrystals, if they are close enough. The highly energetic excitation can then create multiple excitons in different adjacent nanocrystals. The spatial extent of these wavefunctions is much smaller, consequently Auger recombination becomes less likely and in turn radiative recombination is enhanced. This model thus offers an explanation for the radiative character of MEG in our system.

To substantiate our proposed model, we have also measured a highly diluted dispersion of $\mathrm{CsPbBr}_{3} \mathrm{NCs}$ on substrate (see Supplementary Fig. 7). While we could measure linearly excited PL from these NCs, we did not observe the MPE-MEG resonances observed for a dense array of NCs. This further supports the idea 

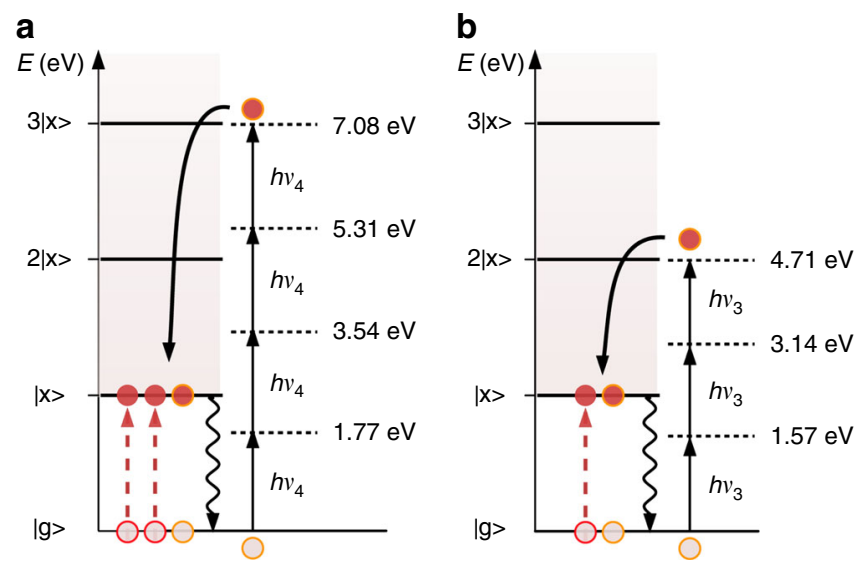

Fig. 4 Energy diagram of the resonances between MPE and MEG in $\mathrm{CsPbr}_{3} \mathrm{NCs}$. Photo-excitation at $3 E_{\mathrm{x}}$ (a) and $2 E_{\mathrm{x}}$ (b) and subsequent generation three (a) and two (b) excitons via multiple photon excitation processes with photons of energies $h \nu_{4}$ and $h \nu_{3}$, respectively

that a dense arrangement of the NCs is crucial for observing this phenomenon and for suppressing non-radiative Auger recombination. Further validity is obtained by repeating the investigations on larger $\mathrm{CsPbBr}_{3}$ nanocubes with edge sizes on the order of 100 nm (Supplementary Fig. 8). While we observe an enhanced PL near the expected resonance positions, the resonances themselves-if present-are significantly weaker and strongly broadened. This is to be expected, as Auger recombination will not be similarly suppressed as in the case of the smaller close-packed NCs.

In conclusion, we have shown that perovskite NCs exhibit interband PL emission when excited with photons with belowband-gap energies over a wide range of energies. Our findings show that multiple excitons can be generated in non-quantum-confined perovskite NCs by the excitation with multiple photons and that the system exhibits resonances for specific values of the excitation wavelength. These resonances occur when the total energy of a number of exciting photons is equal to the energy necessary to create multiple excitons. Owing to the presence of resonances, the photogeneration together with the radiative recombination of excitons are highly enhanced, while the non-radiative Auger recombination is suppressed due to the spatial arrangement of the NCs on substrate. Therefore, high-order MPE can be used as an efficient pathway for the creation of multiple excitons in non-quantum-confined perovskite nanocrystals. Moreover, having observed the presence of MEG-MPE resonances in different perovskite NCs, we can envision this process also occurring in other semiconducting nanocrystals even with sizes greater than the quantum dot restriction. This could open new pathways for various energy conversion systems exploiting high light intensities.

\section{Methods}

Materials. The synthesis of $\mathrm{CsPbBr}_{3}$ NCs was carried out by ultrasonication of precursors in octadecene solvent mixed with oleylamine and oleic acid ligands, as reported previously by our group ${ }^{26}$. A detailed description of the synthesis techniques can be found in the Supplementary Note 1.

Experimental setup. The excitation for the MPE-PL measurements was provided by a SuperK EXTREME supercontinuum white light laser focused by a $100 \times$ magnification to the specimen. More information about the experimental setup is in the Supplementary Note 2. All the measurements were conducted in normal atmosphere and at room temperature.

Feasibility studies. An estimation of the efficiency of the proposed mechanism for solar applications can be found in the Supplementary Note 3.
Data availability. The data that support the findings of this study are available from the corresponding authors on reasonable request.

Received: 28 August 2017 Accepted: 22 March 2018

Published online: 17 April 2018

\section{References}

1. He, G. S., Tan, L. S., Zheng, Q. \& Prasad, P. N. Multiphoton absorbing materials: Molecular designs, characterizations, and applications. Chem. Rev. 1080, 1245-1330 (2008).

2. Trupke, T., Green, M. A. \& Würfel, P. Improving solar cell efficiencies by down-conversion of high-energy photons. J. Appl. Phys. 920, 1668-1674 (2002).

3. Schaller, R. D., Agranovich, V. M. \& Klimov., V. I. High-efficiency carrier multiplication through direct photogeneration of multi-excitons via virtual single-exciton states. Nat. Phys. 10, 189-194 (2005).

4. Klimov, V. I. Mechanisms for photogeneration and recombination of multiexcitons in semiconductor nanocrystals: Implications for lasing and solar energy conversion. J. Phys. Chem. B 1100, 16827-16845 (2006).

5. Kamat, P. V. Quantum dot solar cells. Semiconductor nanocrystals as light harvesters. J. Phys. Chem. C 1120, 18737-18753 (2008).

6. Schaller, R. D. \& Klimov, V. I. High efficiency carrier multiplication in PbSe nanocrystals: Implications for solar energy conversion. Phys. Rev. Lett. 920, 186601-186601 (2004).

7. Ellingson, R. J. et al. Highly efficient multiple excition generation in colloidal PbSe and PbS quantum dots. Nano Lett. 5, 865-871 (2005).

8. Beard, M. C. et al. Multiple exciton generation in colloidal silicon nanocrystals. Nano Lett. 70, 2506-2512 (2007).

9. Pijpers, J. J. H. et al. Carrier multiplication and its reduction by photodoping in colloidal inas quantum dots carrier multiplication and its reduction by photodoping in colloidal InAs quantum dots. J. Phys. Chem. 111, 4146-4152 (2007).

10. Nozik, A. J. Multiple exciton generation in semiconductor quantum dots. J. Phys. Chem. Lett. 457, 3-11 (2008).

11. Beard, M. C. Multiple exciton generation in semiconductor quantum dots. $J$ Phys. Chem. Lett. 20, 1282-1288 (2011).

12. Kumar, M. et al. Hot exciton cooling and multiple exciton generation in $\mathrm{PbSe}$ quantum dots. Phys. Chem. Chem. Phys. 18, 31107-31114 (2016).

13. Swarnkar, A., et al. Quantum dot-induced phase stabilization of $\alpha-\mathrm{CsPb}_{3}$ perovskite for high-efficiency photovoltaics. Science 354, 92-95 (2016).

14. Akkerman, Q. A. et al. Strongly emissive perovskite nanocrystal inks for highvoltage solar cells. Nat. Energy 2, 1-7 (2016).

15. Protesescu, L. et al. Nanocrystals of cesium lead halide perovskites $\left(\mathrm{CsPbX}_{3}, \mathrm{X}=\mathrm{Cl}, \mathrm{Br}\right.$, and $\left.\mathrm{I}\right)$ : novel optoelectronic materials showing bright emission with wide color gamut. Nano Lett. 15, 3692-3696 (2015).

16. Walters, G. et al. Two-photon absorption in organometallic bromide perovskites. ACS Nano 90, 9340-9346 (2015).

17. Wang, Y. et al. Nonlinear absorption and low-threshold multiphoton pumped stimulated emission from all-inorganic perovskite nanocrystals. Nano Lett. 160, 448-453 (2016).

18. Wei, K. et al. Temperature-dependent excitonic photoluminescence excited by two-photon absorption in perovskite $\mathrm{CsPbBr}_{3}$ quantum dots. Opt. Lett. 410, 16-19 (2016).

19. Zhang, R. et al. Nonlinear optical response of organic-inorganic halide perovskites. ACS Photonics 3, 371-377 (2016).

20. Lu, W. G. et al. Nonlinear optical properties of colloidal $\mathrm{CH}_{3} \mathrm{NH}_{3} \mathrm{PbBr}_{3}$ and $\mathrm{CsPbBr}_{3}$ quantum dots: a comparison study using Z-scan technique. Adv. Opt. Mater. 40, 1732-1737 (2016).

21. Gu, Z. et al. Two-photon pumped $\mathrm{CH}_{3} \mathrm{NH}_{3} \mathrm{PbBr}_{3}$ perovskite microwire lasers Adv. Opt. Mater. 40, 472-479 (2016).

22. $\mathrm{Li}$, J. et al. Two-photon absorption and emission in $\mathrm{CsPb}(\mathrm{Br} / \mathrm{I})_{3}$ cesium lead halide perovskite quantum dots. CrystEngComm 180, 7945-7949 (2016).

23. Makarov, N. S. et al. Spectral and dynamical properties of single excitons, biexcitons, and trions in cesium-lead-halide perovskite quantum dots. Nano Lett. 160, 2349-2362 (2016).

24. de Jong, E. M. L. D. et al. Multiexciton lifetime in all-inorganic $\mathrm{CsPbBr}_{3}$ perovskite nanocrystals. J. Phys. Chem. C. 121, 1941-1947 (2016).

25. Aneesh, J. et al. Ultrafast exciton dynamics in colloidal $\mathrm{CsPbBr}$. perovskite nanocrystals: bi-exciton effect and auger recombination. J. Phys. Chem. C. 121, 4737-4739 (2017)

26. Tong, Y. et al. Highly luminescent cesium lead halide perovskite nanocrystals with tunable composition and thickness by ultrasonication. Angew. Chem. Int. Ed. 550, 13887-13892 (2016).

27. Sichert, J. A. et al. Quantum size effect in organometal halide perovskite nanoplatelets. Nano Lett. 150, 6521-6527 (2015). 
28. Kim, Y.-h et al. Highly efficient light-emitting diodes of colloidal metal-halide perovskite nanocrystals beyond quantum size. ACS Nano 11, 6586-6593 (2017).

29. Trinh, M. T. et al. Direct generation of multiple excitons in adjacent silicon nanocrystals revealed by induced absorption. Nat. Photon. 60, 316-321 (2012).

\section{Acknowledgements}

This work was supported by the Bavarian State Ministry of Science, Research, and Arts through the grant "Solar Technologies go Hybrid (SolTech)", the DFG-project

"LMUexcellent", the EU-project "COMPASS" and the China Scholarship Council (Y.T.).

\section{Author contributions}

J.F. and L.P. suggested below-band-gap experiments on perovskite NCs. A.M. performed most of the optical measurements and the analytical work. Y.T. and L.P. synthesized and characterized the perovskite nanocubes. Ju.F. and E-P.Y. contributed to the optical measurements on $\mathrm{CsPbI}_{3} \mathrm{NCs}$. A.M. wrote the manuscript with inputs from J.F. and A.S. $\mathrm{U}$. All the authors contributed to the discussion.

\section{Additional information}

Supplementary Information accompanies this paper at https://doi.org/10.1038/s41467018-03965-8.
Competing interests: The authors declare no competing interests.

Reprints and permission information is available online at http://npg.nature.com/ reprintsandpermissions/

Publisher's note: Springer Nature remains neutral with regard to jurisdictional claims in published maps and institutional affiliations.

(c) (i) Open Access This article is licensed under a Creative Commons Attribution 4.0 International License, which permits use, sharing, adaptation, distribution and reproduction in any medium or format, as long as you give appropriate credit to the original author(s) and the source, provide a link to the Creative Commons license, and indicate if changes were made. The images or other third party material in this article are included in the article's Creative Commons license, unless indicated otherwise in a credit line to the material. If material is not included in the article's Creative Commons license and your intended use is not permitted by statutory regulation or exceeds the permitted use, you will need to obtain permission directly from the copyright holder. To view a copy of this license, visit http://creativecommons.org/ licenses/by/4.0/.

(C) The Author(s) 2018 